\title{
Factors Associated with Postnatal Care Services Uptake by Young Mothers Aged 15 - 24 Years at Mukono General Hospital, Uganda
}

\author{
Kizito Omona $[\mathrm{PhD}]^{1}{ }^{*}$; Bernadette Nabuuma ${ }^{1}$; Amir Wamala ${ }^{1}$; Doreen Nagawa ${ }^{1}$; \\ Kenedy Bukadu ${ }^{1}$; Alex Lwasa Namaala ${ }^{1}$ \\ ${ }^{1}$ Faculty of Health Sciences, Uganda Martyrs University, Kampala, Uganda \\ Corresponding Author: Dr. Omona Kizito, Lecturer. E-mail: komona@umu.ac.ug, Tel: +256706464873 \\ DOI: 10.47760/cognizance.2021.v01i03.004
}

\begin{abstract}
Introduction: Postnatal period is the time shortly after birth of the baby and extending up to six weeks. This period is recognized as very critical for survival of both the mother and the baby. Recent guidelines recommend four postnatal care contacts with skilled health personnel for the health and well-being of mother and newborn.

Objective: The purpose of this study was to establish the factors associated with uptake of Postnatal Care (PNC) Services by young mothers (15 - 24 years) at Mukono General Hospital, Mukono District.

Method: A cross-sectional analytical study design utilizing a quantitative approach was employed. Mothers (325) who had given birth in the last six months were systematically enrolled into the study. Interviewer-administered questionnaires were used for data collection. Data was coded and entered into the computer using SPSS version 22 with programmed quality control checks. Frequency tables and graphs were used to describe the study variables. Univariate, bivariate and multivariate logistic regression analysis methods were used to identify factors associated with uptake of PNC services. $P$ value of $<0.05$ with $95 \%$ CI were used to declare statistical significance.

Results: From the 325 respondents' data analyzed, uptake of PNC services was at $78.8 \%$. Being selfemployed, $(P=0.003)$, employed $(P=0.003)$, husband employed $(P=0.003)$, not receiving $P N C$ services privately $(P=0.000)$, spending more than 5000 shillings on $P N C(P=0.004)$ and being married $(P=0.004)$ were independently associated with uptake of PNC services. The study revealed that the level of PNC uptake was moderate and its utilization is influenced by being employed, husband being employed, not having to receive PNC services privately, spending less than 2000 shillings on PNC, and being married.

Conclusion: This study has shown that mothers whose partners were working either formally or informally utilized postnatal care services more than mothers whose partners were unemployed. Hence forth, government/partners should consider empowering citizens especially women economically through job creation initiatives, among others
\end{abstract}

Keywords: PNC services utilization/uptake, Associated Factors, Young mothers, Mukono General Hospital 


\section{INTRODUCTION \\ 1.1 Background of the study}

The Postnatal period is the time shortly after birth of the baby and extending up to six weeks (1). This period is recognized as very critical for survival of both the mother and the new born (2). Recent guidelines recommend four postnatal care visits or contacts with skilled health personnel for the health and well-being of mother and newborn: on day 1 (within 24 hours after delivery), on day 3 ( $48-72$ hours after birth), between days $7-14$ and at six weeks (1). Mothers are at a very significant risk of mortality from bleeding and infection while preterm birth, asphyxia and severe infections pose greatest risk to the lives of the newborns (2). More than $90 \%$ of all maternal deaths occur in low and lower middle income countries (3). The majority of maternal and infant deaths occur within the first four weeks after child birth (4). Close to 7000 newborns die every day with almost one third dying within 24 hours of birth and about 75\% dying within their first week of life (5). While more than two thirds of neonatal deaths arise from complications of preterm birth, asphyxia and severe infections, inappropriate feeding and cultural practices during the postnatal period continue to pose more risks to the lives of the newborn (4).

Maternal complications following child birth like chronic pain, impaired mobility, damage to the reproductive system and infertility are more common and aggravated in developing countries (6). Additionally, of the maternal and neonatal deaths that occur globally, more than $90 \%$ occur in low and lower-middle income countries $(3,4)$.

In rural Myanmar, just 25\% of women utilized a full postnatal care service package (7). A secondary education level or higher, higher income level, male involvement, low birth order and being aware of postnatal danger signs influenced utilization of this service by the mothers (7). Similarly, in Bangladesh, $33 \%$ of young mothers received postpartum care and a very small proportion of them (between 16.6 and 25.5\%) sought this care from medically trained personnel within 48 hours after delivery (8). In tandem with other studies in Nepal, a mere $26.5 \%$ of mothers was reported to have attended at least one postnatal visit (9).

In Nigeria, slightly more than half of women of reproductive age had at least four ANC visits during their last pregnancies, of which $37.3 \%$ delivered in a health facility and $28.9 \%$ of newborn babies received postnatal care within two months of delivery (10). Factors that are significantly associated with utilization of postnatal care are formal education, ANC attendance, distance from health facilities and lack of awareness about PNC (11). Women who have antenatal care follow-up and women who deliver at health institutions are more likely to have full PNC visits (12). Abraha et al (2019) contrary to other studies found that proximity of women to health facilities lowered the likelihood of completing PNC visits. A community based cross-sectional survey in Northern Shoa Zone, Ethiopia found a low prevalence $(28.4 \%)$ of full postnatal care service utilization among women of reproductive age. The mode of delivery, number of children and level of education were significantly associated with postnatal care service uptake (13).

In South Africa, more than half (59.6\%) of mothers and their newborns received three postnatal care visits in the first six weeks following delivery (14). At least four ANC visits, an initial ANC visit in the first trimester, delivery in a health facility and exposure to HIV were significantly associated with uptake of PNC. Moreover, mothers who were HIV positive had more PNC contacts compared to their non-exposed counterparts (14).

In Zambia, utilization of PNC within 48 hours of delivery was predicted by at least one ANC visit and skilled birth attendance (15). In addition, few (34.6\%) postpartum women in Democratic Republic of Congo received PNC in the 42 days after child birth even though a vast majority of them attended at least one ANC visit and gave birth at a health facility under 

pregnancies and those who did not get complications of child birth were less likely to use ANC and PNC (16).

In rural Tanzania, the district of residence, ethnic group, a wanted pregnancy, ANC attendance, place of delivery, and any incidence of the newborn predicted utilization of PNC(17). Unwanted pregnancies reduced mothers' chances of attending PNC services while sick newborns increased the likelihood of receiving PNC in the first 4 weeks after child birth (17). However, compared with results from other studies, in this area mothers who delivered at a health facility did not attend PNC services as much as those who delivered outside a facility (17).

In Karamoja sub region, postnatal care coverage was estimated at $31.3 \%$ although the service was available in $65 \%$ of health facilities in the region (18). Secondary and higher education, level of income and urban residence increased utilization of maternal health services encompassing prenatal, delivery and post-delivery care (19). Furthermore, utilization of postnatal care in Luwero area was influenced by mothers' parity, marital status, cost of PNC services and waiting times at health facilities (20). In the eastern district of Soroti, information about PNC schedules and formal employment were significantly associated with PNC attendance while women who delivered from private health facilities were more likely attend PNC than those who delivered at public facilities (21).

Young mothers aged $15-24$ years fall in the venerable age group considered to be most at risk for HIV and unwanted pregnancy (22). These young mothers $15-24$ years have a significantly higher risk of severe morbidity and complications of child birth compared to their counterparts above 25 years of age (23).

Over the years, Uganda has made remarkable progress in improving birth outcomes for both mothers and newborns for example $97 \%$ of all women receive ANC from skilled health personnel, $73 \%$ of live births occur in health facilities with $74 \%$ skilled birth attendance but just slightly more than half of women and newborns receive a postnatal check with 48 hours of delivery (24). No wonder, Uganda's maternal mortality ratio estimated at 336 maternal deaths per 100,000 live births is still unacceptably high (24).

Postnatal care provides a window of opportunity to avert possible deaths and morbidities for mothers and newborns since majority of these occur after delivery (11). But unfortunately, often times postnatal care is missed by mothers and newborns a situation that makes their health and survival hang in balance (13). While more than 700 women deliver at Mukono General Hospital every month (25), there is no study to our knowledge that has assessed factors associated with uptake of PNC services by young mothers aged 15-24 years at this health facility. It is against this background that this study assessed the factors associated with uptake of PNC services by young mothers (15-24 years) at Mukono General Hospital.

\subsection{Broad objective}

The purpose of this study was to establish the factors associated with uptake of Postnatal Care (PNC) Services by young mothers (15 - 24 years) at Mukono General Hospital, Mukono District.

\subsection{Specific objectives}

(1) To establish the distribution of timing for uptake PNC services by young mothers of aged 15-24years at Mokono General Hospital

(2) To determine the socio-demographic factors associated with uptake of Postnatal Care (PNC) by young mothers of aged 15-24years at Mokono General Hospital 
cognizancejournal.com

Kizito Omona et al, Cognizance Journal of Multidisciplinary Studies, Vol.1, Issue.3, March 2021, pg. 45-57

(3) To determine the socio-economic factors associated with uptake of PNC services by young mothers of aged 15-24years at Mokono General Hospital

(4) To determine the health institutional factors associated with uptake of PNC services by young mothers of aged 15-24years at Mokono General Hospital

\section{METHODs}

\subsection{Study design}

The study employed a cross-sectional analytical study design to generate data from selected samples using a questionnaire.

\subsection{Study population}

Mothers aged 15-24years attending health clinics at Mukono General Hospital who met the inclusion criteria were selected using a systematic random sampling method until the required sample size was obtained.

\subsection{Inclusion and Exclusion Criteria}

\subsubsection{Inclusion criteria}

Mothers, aged 15-24years old attending health clinics in the health facility were included.

\subsubsection{Exclusion criteria}

Mothers aged less than 15years or older than 24years old attending health clinics in the health facility were excluded.

\section{4 Sample size estimation}

Based on the number of mothers who deliver at Mukono General Hospital, in a six weeks' period approximately 1125 women are expected to deliver at this facility (25). This population of mothers provided the sampling frame from which sample size was calculated basing on Slovin's formula/sampling technique for sample size determination in a finite population.

$\boldsymbol{n}=\frac{N}{\left(1+N e^{2}\right)}$

Where;

$\boldsymbol{n}=$ the sample size

$\mathrm{N}=$ Sampling frame (1125 mothers delivering in 6 weeks at Mukono hospital)

$\mathrm{e}=$ margin of error set at $5 \%(0.05)$

Therefore; $\quad \boldsymbol{n}=\frac{1125}{\left(1+1125 \times 0.05^{2}\right)}=295.08=295$

Considering a non-response rate of $10 \%(295 \times 10 \%=29.5)$

Therefore, $\mathrm{n}=295+29.5=324.5$ which is approximately 325 respondents

The study involved a sample of 325 respondents who were selected using systematic random sampling. 


\subsection{Sampling procedure}

The researchers used a systematic random sampling technique to acquire the desired respondents for the study. The mothers attending health clinics at Mukono General Hospital who met the inclusion criteria were selected using a systematic random sampling method until the required sample size was obtained.

The sampling interval, $\mathrm{K}=\frac{\text { Sampling frame }(N)}{\text { Sample Size }(n)}$

Therefore, $\mathrm{K}=\frac{1125}{325}=3.46$, approximately 4clients. Hence, using systematic random sampling the researchers were able to select every $4^{\text {th }}$ mother from the clients visiting the clinics per day.

The respondents were briefed about the study and questionnaires administered by research assistants to respondents who consented to participate in the study.

\subsection{Data collection tools}

An interviewer-administered structured questionnaire both in English and translated version in Luganda were used for data collection. It contained both open-ended and closed-ended questions.

\subsection{Data management}

Data processing was done by editing, coding statistically and tabulating, and after the processing, data was entered into Statistical Package for Social Science (SPSS) V.20 for further analysis. Univariate analysis was done using descriptive frequency tests and then summarized and presented in tables, and bar graphs. Descriptive statistics like frequency counts, percentage distributions, mean and standard deviation were displayed.

Bi-variate analysis was also done using cross tabulations in chi-square and odds risk assessment test for the relationship between the independent and dependent variables as stated above. Hence the Chi square test was the major statistical test for the result before conclusion. Binary regression was used at multivariate level to generate the odd ratios (OR)

\subsection{Ethical considerations}

Uganda Martyrs University Faculty of Health Sciences Ethics committee approved the study. Approval letter was presented to the authorities of Mukono General Hospital asking for permission to carry out the study. The respondents provided informed consent before enrolment into the study and they had an option to withdraw from the study at any time.

Confidentiality was maximized and thus, findings were presented in a generalized manner without exposing personal participant information.

\section{RESULTS}

\subsection{Socio-demographic Characteristics of Respondents}

A total of 325 mothers attending Mukono General Hospital clinics consented to participate in the study and the response rate was $100 \%$. 
Table 1: Demographic Characteristics of Respondents

\begin{tabular}{|c|c|c|c|}
\hline Variables & Category & Frequency (n) & Percentage $(\%)$ \\
\hline 1) Age Group & $\begin{array}{l}15-19 \text { Years } \\
20-24 \text { Years }\end{array}$ & $\begin{array}{l}88 \\
237\end{array}$ & $\begin{array}{l}27.1 \\
72.9\end{array}$ \\
\hline 2) Delivery place & $\begin{array}{l}\text { Hospital } \\
\text { Home } \\
\text { Other }\end{array}$ & $\begin{array}{l}283 \\
18 \\
24\end{array}$ & $\begin{array}{l}87.1 \\
5.5 \\
7.4\end{array}$ \\
\hline 3) Method of delivery & $\begin{array}{l}\text { Normal vaginal delivery } \\
\text { Caesarean section } \\
\text { Assisted vaginal delivery }\end{array}$ & $\begin{array}{l}224 \\
89 \\
12\end{array}$ & $\begin{array}{l}68.9 \\
27.4 \\
3.7\end{array}$ \\
\hline $\begin{array}{l}\text { 4) Knowledge of PNC services you } \\
\text { are supposed to receive after } \\
\text { delivery }\end{array}$ & $\begin{array}{l}\text { Yes } \\
\text { No }\end{array}$ & $\begin{array}{l}253 \\
72\end{array}$ & $\begin{array}{l}77.8 \\
22.2\end{array}$ \\
\hline 5) Reasons for not attending PNC & $\begin{array}{l}\text { Attending to other family } \\
\text { matters } \\
\text { Not aware } \\
\text { It is expensive } \\
\text { Beliefs } \\
\text { Do not stay in the area }\end{array}$ & $\begin{array}{l}17 \\
45 \\
7 \\
7 \\
1\end{array}$ & $\begin{array}{l}24.6 \\
65.2 \\
10.2 \\
10.2 \\
1.5\end{array}$ \\
\hline 6) Level of education & $\begin{array}{l}\text { Never gone to school } \\
\text { Preprimary } \\
\text { Secondary } \\
\text { Tertiary } \\
\text { Vocational }\end{array}$ & $\begin{array}{l}92 \\
59 \\
73 \\
82 \\
19\end{array}$ & $\begin{array}{l}28.3 \\
18.2 \\
22.5 \\
25.2 \\
5.8\end{array}$ \\
\hline 7) Employment status & $\begin{array}{l}\text { Un-employed } \\
\text { Self-employed } \\
\text { Employed }\end{array}$ & $\begin{array}{l}120 \\
118 \\
87\end{array}$ & $\begin{array}{l}36.9 \\
36.3 \\
26.8\end{array}$ \\
\hline 8) Husband occupation & $\begin{array}{l}\text { Un-employed } \\
\text { Self-employed } \\
\text { Employed }\end{array}$ & $\begin{array}{l}68 \\
130 \\
127\end{array}$ & $\begin{array}{l}20.9 \\
40.0 \\
39.1\end{array}$ \\
\hline $\begin{array}{l}\text { 9) Average Income level in a } \\
\text { month }\end{array}$ & $\begin{array}{l}<100,000 \\
100,000-200,000 \\
200,001-400,000 \\
400,001-1,000,000\end{array}$ & $\begin{array}{l}139 \\
80 \\
65 \\
41\end{array}$ & $\begin{array}{l}42.8 \\
24.6 \\
20.0 \\
12.6\end{array}$ \\
\hline 10) Religion & $\begin{array}{l}\text { Roman Catholic } \\
\text { Anglican } \\
\text { Moslem } \\
\text { Pentecostal } \\
\text { Other }\end{array}$ & $\begin{array}{l}74 \\
103 \\
38 \\
80 \\
30\end{array}$ & $\begin{array}{l}22.8 \\
31.7 \\
11.7 \\
24.6 \\
9.2\end{array}$ \\
\hline $\begin{array}{l}\text { 11) Cultural beliefs / practices } \\
\text { that influence seeking for PNC } \\
\text { services }\end{array}$ & $\begin{array}{l}\text { Yes } \\
\text { No }\end{array}$ & $\begin{array}{l}87 \\
238\end{array}$ & $\begin{array}{l}26.8 \\
73.2\end{array}$ \\
\hline
\end{tabular}

From table 1 above, the majority (72.9\%) of the respondents were in the age group of 20-24 years. Most $(87.1 \%)$ of them delivered in the hospitals and $68.9 \%$ had normal vaginal delivery. Majority of the respondents $(28.3 \%$ ) had no formal education while $25.2 \%$ had attained tertiary education. $36.9 \%$ of the respondents were unemployed while $36.3 \%$ were self-employed and four in every ten (40\%) of the respondents' husbands were self-employed. Less than half $(42.8 \%)$ of the respondents had average monthly income of $<100,000$ shilling. 
Regarding religion, majority $31.7 \%$ of the respondents were Anglicans and $73.2 \%$ of the respondents had no cultural beliefs or practices that influence seeking PNC services.

\subsection{Distribution of Timing for Uptake of PNC Services Among Mothers}

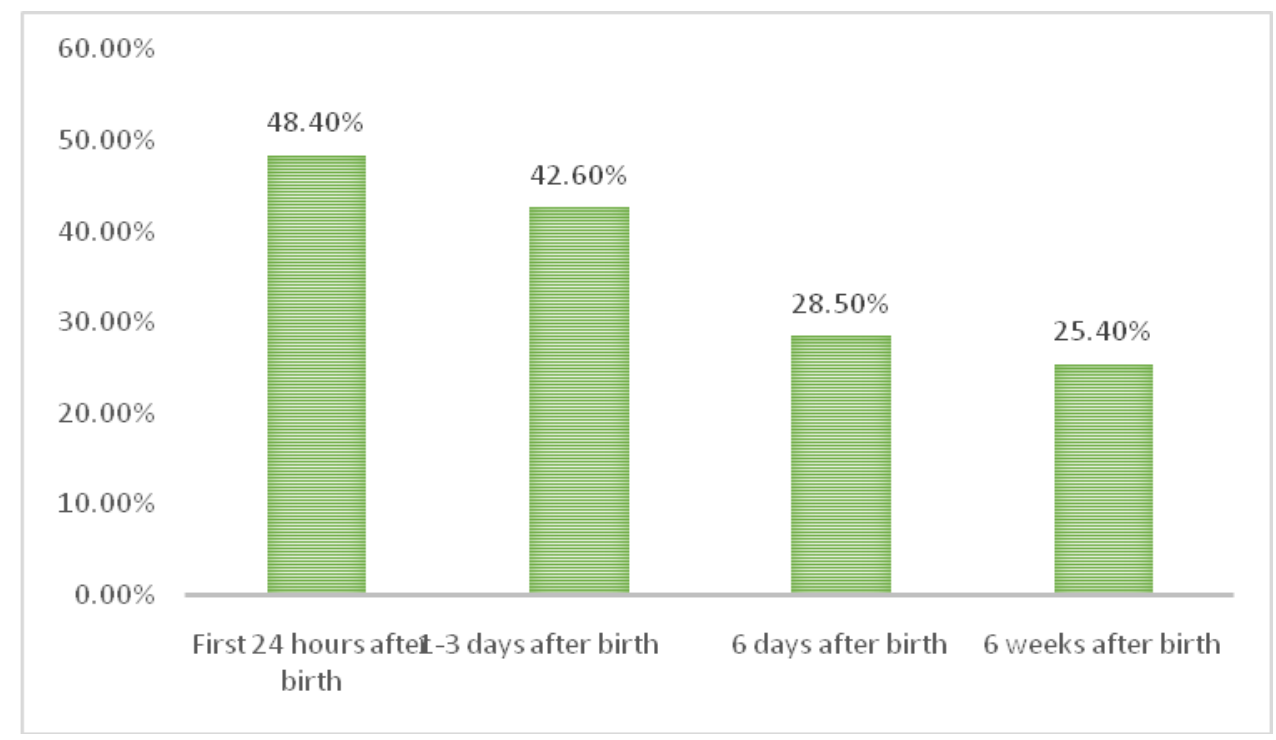

Figure 1: Distribution of timing for PNC Among Mothers

From figure 1, among those respondent mothers who received PNC services, $48.4 \%$ of them received it in the first 24 hours after birth compared to $42.6 \%$ who received it in 1-3 days after birth. Fewer mothers (25.4\%), received PNC 6 weeks after birth. The main reasons for attending PNC services were because the baby needed immunization (44.9\%) and because the midwife/doctor recommended it $(34.8 \%)$.

\subsection{Socio-demographic Factors Associated with Uptake of Postnatal Care (PNC)}

Bivariate analysis was run and the results are shown in table 2 below.

Table 2: Socio-demographic Factors Associated with Uptake of Postnatal Care (PNC)

\begin{tabular}{|c|c|c|c|c|c|}
\hline \multirow[t]{2}{*}{ Variables } & \multirow[t]{2}{*}{ Category } & \multicolumn{2}{|c|}{ Attended PNC } & \multirow{2}{*}{$\begin{array}{l}\text { Chi } \\
\left(\chi^{2}\right)\end{array}$} & \multirow[t]{2}{*}{ p-value } \\
\hline & & Yes $(\%)$ & No $(\%)$ & & \\
\hline \multirow[t]{2}{*}{ 1) Age Group } & $15-19$ Years & $68(26.6 \%)$ & $20(29.0 \%)$ & 0.162 & .688 \\
\hline & $20-24$ Years & $188(73.4 \%)$ & $49(71.0 \%)$ & $\mathrm{Df}=1$ & \\
\hline \multirow[t]{3}{*}{ 2) Delivery place } & Hospital & $225(87.9 \%)$ & $58(84.1 \%)$ & 22.418 & $.009^{*}$ \\
\hline & Home & $15(5.9 \%)$ & $3(4.3 \%)$ & $\mathrm{Df}=2$ & \\
\hline & Other & $16(6.2 \%)$ & $8(11.6 \%)$ & & \\
\hline \multirow[t]{3}{*}{ 3) Method of delivery } & Normal vaginal delivery & $173(67.6 \%)$ & $51(73.9 \%)$ & 1.029 & .598 \\
\hline & Caesarean section & $73(28.5 \%)$ & $16(23.2 \%)$ & $\mathrm{Df}=2$ & \\
\hline & Assisted vaginal delivery & $10(3.9 \%)$ & $2(2.9 \%)$ & & \\
\hline \multirow{2}{*}{$\begin{array}{l}\text { 4) Knowledge of PNC } \\
\text { services you are supposed to } \\
\text { receive after delivery }\end{array}$} & Yes & $200(78.1 \%)$ & $53(76.8 \%)$ & .054 & .816 \\
\hline & No & $56(21.9 \%)$ & $16(23.2 \%)$ & $\mathrm{Df}=1$ & \\
\hline
\end{tabular}


cognizancejournal.com

Kizito Omona et al, Cognizance Journal of Multidisciplinary Studies, Vol.1, Issue.3, March 2021, pg. 45-57

\begin{tabular}{|c|c|c|c|c|c|}
\hline 5) Marital Status & $\begin{array}{l}\text { Single } \\
\text { Married } \\
\text { Divorced/separated } \\
\text { Widowed }\end{array}$ & $\begin{array}{l}38(14.8 \%) \\
195(76.2 \%) \\
9(3.5 \%) \\
14(5.5 \%)\end{array}$ & $\begin{array}{l}44(63.8 \%) \\
19(27.5 \%) \\
5(7.2 \%) \\
1(1.4 \%)\end{array}$ & $\begin{array}{l}74.745 \\
\text { Df }=3\end{array}$ & $.000 *$ \\
\hline $\begin{array}{l}\text { 6) Number of people in the } \\
\text { household }\end{array}$ & $\begin{array}{l}\text { Less than } 5 \text { people } \\
5-10 \text { people } \\
\text { More than } 10 \text { people }\end{array}$ & $\begin{array}{l}165(64.5 \%) \\
83(32.4 \%) \\
8(3.1 \%)\end{array}$ & $\begin{array}{l}57(82.6 \%) \\
12(17.4 \%) \\
0(0.0 \%)\end{array}$ & $\begin{array}{l}8.980 \\
\mathrm{Df}=2\end{array}$ & $.011 *$ \\
\hline $\begin{array}{l}\text { 7) Number of pregnancies } \\
\text { ever had }\end{array}$ & $\begin{array}{l}1-2 \\
3-4 \\
4 \text { and above }\end{array}$ & $\begin{array}{l}124(48.4 \%) \\
79(30.9 \%) \\
53(20.7 \%)\end{array}$ & $\begin{array}{l}43(62.3 \%) \\
23(33.3 \%) \\
3(4.3 \%)\end{array}$ & $\begin{array}{l}10.599 \\
\mathrm{Df}=2\end{array}$ & $.014 *$ \\
\hline $\begin{array}{l}\text { 8) No. of Live biological } \\
\text { children }\end{array}$ & $\begin{array}{l}1-2 \\
3-4 \\
\text { Above } 4\end{array}$ & $\begin{array}{l}163(63.7 \%) \\
69(27.0 \%) \\
24(9.4 \%)\end{array}$ & $\begin{array}{l}56(81.2 \%) \\
12(17.4 \%) \\
1(1.4 \%)\end{array}$ & $\begin{array}{l}8.899 \\
\mathrm{Df}=2\end{array}$ & $.012 *$ \\
\hline $\begin{array}{l}\text { 9) Received any support } \\
\text { from your spouse or } \\
\text { relative }\end{array}$ & $\begin{array}{l}\text { Yes } \\
\text { No }\end{array}$ & $\begin{array}{l}221(86.3 \%) \\
35(13.7 \%)\end{array}$ & $\begin{array}{l}40(58.0 \%) \\
29(42.0 \%)\end{array}$ & $\begin{array}{l}27.636 \\
\mathrm{Df}=1\end{array}$ & $.000 *$ \\
\hline $\begin{array}{l}\text { 10) Took any substance or } \\
\text { drug }\end{array}$ & $\begin{array}{l}\text { Yes } \\
\text { No }\end{array}$ & $\begin{array}{l}52(20.3 \%) \\
204(79.7 \%)\end{array}$ & $\begin{array}{l}11(15.9 \%) \\
58(84.1 \%)\end{array}$ & $\begin{array}{l}.664 \\
\mathrm{Df}=1\end{array}$ & .415 \\
\hline
\end{tabular}

$\mathbf{D f}=$ Degree of freedom

From table 2, the only demographic factor associated with uptake of PNC services was place of delivery $\left(\chi^{2}=22.418, p=0.009\right)$.

Among the family related factors, marital status $\left(\chi^{2}=74.745, \mathrm{p}=0.000\right)$, number of people living in the households $\left(\chi^{2}=10.599, \mathrm{p}=0.014\right)$, number of biological children alive $\left(\chi^{2}=8.899\right.$, $\mathrm{p}=0.012)$ and receiving some form of support from spouse or relatives $\left(\chi^{2}=27.636, \mathrm{p}=0.000\right)$ were significantly associated with uptake of postnatal services among young mothers.

3.4 Socio-economic Factors Associated with Uptake of PNC

Bivariate analysis was run and the results shown in table below.

Table 3: Socio-economic Factors Associated with Uptake of PNC

\begin{tabular}{|c|c|c|c|c|c|}
\hline \multirow[t]{2}{*}{ Variables } & \multirow[t]{2}{*}{ Category } & \multicolumn{2}{|c|}{ Attended PNC } & \multirow{2}{*}{$\begin{array}{l}\text { Chi } \\
\operatorname{Sq}\left(\chi^{2}\right)\end{array}$} & \multirow[t]{2}{*}{ p-value } \\
\hline & & Yes $(\%)$ & No $(\%)$ & & \\
\hline \multirow[t]{5}{*}{ Level of education } & Never gone to school & $41(16.0 \%)$ & $51(73.9 \%)$ & 96.996 & $.000^{*}$ \\
\hline & Preprimary & $55(21.5 \%)$ & $4(5.8 \%)$ & $\mathrm{Df}=4$ & \\
\hline & Secondary & $60(23.4 \%)$ & $13(18.8 \%)$ & & \\
\hline & Tertiary & $81(31.6 \%)$ & $1(1.4 \%)$ & & \\
\hline & Vocational & $19(7.4 \%)$ & $0(0.0 \%)$ & & \\
\hline \multirow[t]{3}{*}{ Employment status } & Un-employed & $71(27.7 \%)$ & $49(71.0 \%)$ & 48.739 & $.000 *$ \\
\hline & Self-employed & $100(39.1 \%)$ & $18(26.1 \%)$ & $\mathrm{Df}=2$ & \\
\hline & Employed & $85(33.2 \%)$ & $2(2.9 \%)$ & & \\
\hline \multirow[t]{3}{*}{ Husband occupation } & Un-employed & $24(9.4 \%)$ & $44(63.8 \%)$ & 10.222 & $.000 *$ \\
\hline & Self-employed & $110(43.0 \%)$ & $20(29.0 \%)$ & $\mathrm{Df}=2$ & \\
\hline & Employed & $122(47.7 \%)$ & $5(7.2 \%)$ & & \\
\hline \multirow{4}{*}{$\begin{array}{l}\text { Average Income level in } \\
\text { a month }\end{array}$} & $<100,000$ & $88(34.4 \%)$ & $51(73.9 \%)$ & 43.798 & $.000 *$ \\
\hline & $100,000-200,000$ & $64(25.0 \%)$ & $16(23.2 \%)$ & $\mathrm{Df}=3$ & \\
\hline & $200,001-400,000$ & $63(24.6 \%)$ & $2(2.9 \%)$ & & \\
\hline & $400,001-1,000,000$ & $41(16.0 \%)$ & $0(.0 \%)$ & & \\
\hline \multirow[t]{5}{*}{ Religion } & Roman Catholic & $59(23.0 \%)$ & $15(21.7 \%)$ & .627 & .960 \\
\hline & Anglican & $83(32.4 \%)$ & $20(29.0 \%)$ & $\mathrm{Df}=4$ & \\
\hline & Moslem & $30(11.7 \%)$ & $8(11.6 \%)$ & & \\
\hline & Pentecostal & $61(23.8 \%)$ & $19(27.5 \%)$ & & \\
\hline & Other & $23(9.0 \%)$ & $7(10.1 \%)$ & & \\
\hline
\end{tabular}


cognizancejournal.com

Kizito Omona et al, Cognizance Journal of Multidisciplinary Studies, Vol.1, Issue.3, March 2021, pg. 45-57

\begin{tabular}{lllllll}
\hline Cultural & beliefs $/$ & Yes & $70(27.3 \%)$ & $17(24.6 \%)$ & .203 & .652 \\
practices that influence & No & $186(72.7 \%)$ & $52(75.4 \%)$ & Df=1 & \\
seeking for PNC & & & & & \\
services & & & & & \\
\hline
\end{tabular}

From table 3 , the level of education $\left(\chi^{2}=96.996, \mathrm{p}=0.000\right)$, employment status for the respondents $\left(\chi^{2}=48.739, p\right.$-value of 0.000$)$ and spousal occupation $\left(\chi^{2}=10.222, p=0.000\right)$ and average monthly income $\left(\chi^{2}=43.798, \mathrm{p}=0.000\right)$ were significantly associated with uptake of PNC services.

\subsection{Institutional Factors Associated with PNC Uptake}

Bivariate analysis of institutional factors associated with uptake of postnatal care services by young mothers (15-24 years) was done. See table 4 below.

Table 4: Institutional Factors Associated with PNC Uptake

\begin{tabular}{|c|c|c|c|c|c|}
\hline \multirow[t]{2}{*}{ Variables } & \multirow[t]{2}{*}{ Category } & \multicolumn{2}{|c|}{ Attended PNC } & \multirow{2}{*}{ Chi $\quad \mathrm{Sq}$} & \multirow[t]{2}{*}{ p-value } \\
\hline & & Yes $(\%)$ & No $(\%)$ & & \\
\hline \multirow{2}{*}{$\begin{array}{l}\text { 1) Information about } \\
\text { postnatal care } \\
\text { services during ANC } \\
\text { given }\end{array}$} & Yes & $216(84.4 \%)$ & $58(84.1 \%)$ & .004 & \multirow[t]{2}{*}{.949} \\
\hline & No & $40(15.6 \%)$ & $11(15.9 \%)$ & $\mathrm{Df}=1$ & \\
\hline \multirow{4}{*}{$\begin{array}{l}\text { 2) Information given } \\
\text { by }\end{array}$} & Nurse/Midwife & $197(77.0 \%)$ & $24(34.8 \%)$ & 52.907 & \multirow[t]{4}{*}{$.000 *$} \\
\hline & Doctor & $29(11.3 \%)$ & $24(34.8 \%)$ & $\mathrm{Df}=3$ & \\
\hline & Peer & $23(9.0 \%)$ & $21(30.4 \%)$ & & \\
\hline & Other & $7(2.7 \%)$ & $0(0.0 \%)$ & & \\
\hline \multirow{2}{*}{$\begin{array}{l}\text { 3) Room and } \\
\text { direction labelled }\end{array}$} & Yes & $226(88.3 \%)$ & $60(87.0 \%)$ & .090 & \multirow[t]{2}{*}{.764} \\
\hline & No & $30(11.7 \%)$ & $9(13.0 \%)$ & $\mathrm{Df}=1$ & \\
\hline \multirow{2}{*}{$\begin{array}{l}\text { 4) Get all the PNC } \\
\text { services in one place }\end{array}$} & Yes & $163(63.7 \%)$ & $47(68.1 \%)$ & .469 & \multirow[t]{2}{*}{.493} \\
\hline & No & $93(36.3 \%)$ & $22(31.9 \%)$ & $\mathrm{Df}=1$ & \\
\hline \multirow{2}{*}{$\begin{array}{l}\text { 5) Receive PNC } \\
\text { services privately }\end{array}$} & Yes & $228(89.1 \%)$ & $35(50.7 \%)$ & 51.746 & \multirow[t]{2}{*}{$.000 *$} \\
\hline & No & $28(10.9 \%)$ & $34(49.3 \%)$ & $\mathrm{Df}=1$ & \\
\hline \multirow{4}{*}{$\begin{array}{l}\text { 6) Time spent at the } \\
\text { facility while seeking } \\
\text { PNC services }\end{array}$} & Less than 1-hour & $79(30.9 \%)$ & $17(24.6 \%)$ & 28.215 & \multirow[t]{4}{*}{$.000 *$} \\
\hline & 1 to 2 hours & $99(38.7 \%)$ & $49(71.0 \%)$ & $\mathrm{Df}=3$ & \\
\hline & 3 to 4 hours & $64(25.0 \%)$ & $3(4.3 \%)$ & & \\
\hline & 5 hours and above & $14(5.5 \%)$ & $0(0.0 \%)$ & & \\
\hline \multirow{3}{*}{$\begin{array}{l}\text { 7) Amount spent on } \\
\text { PNC services }\end{array}$} & Less than 2000 & $75(29.3 \%)$ & $68(26.6 \%)$ & 28.479 & \multirow[t]{3}{*}{$.000 *$} \\
\hline & $2000-5000$ & $113(44.1 \%)$ & $26(37.7 \%)$ & $\mathrm{Df}=2$ & \\
\hline & More than 5000 & $68(26.6 \%)$ & $2(2.9 \%)$ & & \\
\hline \multirow{2}{*}{$\begin{array}{l}\text { 8) Own experience of } \\
\text { the maternity } \\
\text { services }\end{array}$} & Good & $156(60.9 \%)$ & $57(82.6 \%)$ & 11.302 & \multirow[t]{2}{*}{$.001 *$} \\
\hline & Bad & $100(39.1 \%)$ & $12(17.4 \%)$ & $\mathrm{Df}=1$ & \\
\hline
\end{tabular}

Table 4 above shows that professional responsible for giving PNC information $\left(\chi^{2}=52.907\right.$, $\mathrm{p}=0.000)$, privacy at PNC clinic $\left(\chi^{2}=51.746, \mathrm{p}=0.000\right)$, waiting time $\left(\chi^{2}=28.215, \mathrm{p}=0.000\right)$, amount spent on PNC services $\left(\chi^{2}=28.479, \mathrm{p}=0.000\right)$, and past experience on maternity services $\left(\chi^{2}=11.302, \mathrm{p}=0.001\right)$ were significantly associated with uptake of PNC services.

Multivariate analysis of factors associated with uptake of PNC services by young mothers (15-24 years) is shown in table 5 below 
Table 5: Multivariate analysis of Factors Associated with Uptake of PNC Services

\begin{tabular}{|c|c|c|c|}
\hline Variable & Category & Adjusted OR (95\% CI) & p-value \\
\hline Employment status & $\begin{array}{l}\text { Un-employed } \\
\text { Self-employed } \\
\text { Employed }\end{array}$ & $\begin{array}{l}1.0 \\
2.205(1.073-4.575) \\
2.022(1.002-5.276)\end{array}$ & $\begin{array}{l}.003 \\
.003\end{array}$ \\
\hline Husband occupation & $\begin{array}{l}\text { Un-employed } \\
\text { Self-employed } \\
\text { Employed }\end{array}$ & $\begin{array}{l}1.0 \\
.367(.116-1.158) \\
4.099(2.021-6.460)\end{array}$ & $\begin{array}{l}.087 \\
.003\end{array}$ \\
\hline $\begin{array}{l}\text { Received PNC } \\
\text { privately }\end{array}$ & $\begin{array}{l}\text { Yes } \\
\text { No }\end{array}$ & $\begin{array}{l}1.0 \\
18.605(4.524-76.510)\end{array}$ & .000 \\
\hline $\begin{array}{l}\text { Amount spent on PNC } \\
\text { services }\end{array}$ & $\begin{array}{l}\text { Less than } 2000 \\
2000-5000 \\
\text { More than } 5000\end{array}$ & $\begin{array}{l}1.0 \\
.568(.171-1.887) \\
.056(.008-.407) \\
\end{array}$ & $\begin{array}{l}.356 \\
.004 \\
\end{array}$ \\
\hline Marital Status & $\begin{array}{l}\text { Single } \\
\text { Married } \\
\text { Divorced/separated } \\
\text { Widowed } \\
\end{array}$ & $\begin{array}{l}1.0 \\
8.142(4.038-17.531) \\
.990(.130-7.544) \\
.102(.004-2.757)\end{array}$ & $\begin{array}{l}.004 \\
.992 \\
.175\end{array}$ \\
\hline
\end{tabular}

Source: Primary data

From table 5, self-employed respondents and those in employment were 2.2times and 2.0 times respectively more likely to use PNC services compared to those who were unemployed.

Respondents whose husbands were employed were 4.1times more likely to use PNC services compared to those whose husbands were unemployed. Respondents who did not receive PNC services privately were 18.6 times more likely to use PNC services compared to those who received PNC services privately.

Respondents who spent more than 5000 shillings in the process if PNC service utilization were 0.056 times less likely to use PNC services compared to those who spent less than 2000 shillings.

Respondents who were married were 8.142 times less likely to use PNC services compared those who were single.

\section{DISCUSSIONS}

Uganda's maternal mortality ratio of 336 maternal deaths per 100,000 live births is still unacceptably high (24), way above the Sustainable development goal target of less than 70 maternal deaths per 100,000 live births for low resource settings (26). The significance of postnatal attendance in helping to avert maternal and perinatal deaths has been noted in past studies $(11,13,23)$.

Our study findings showed that more than three quarters $(78.8 \%)$ of young mothers $15-24$ years received postnatal care in the six weeks following child birth. This finding is higher than the reported national average of 56\% (24).Furthermore this finding is slightly more than double the prevalence of postnatal care among mothers reported by previous studies in different parts of the country (18-21). Compared also past studies in Myanmar (7), Bangladesh, Nepal (8), Nepal (9), Nigeria (10) and Ethiopia (13), our study finding of 78.8\% uptake of PNC is still higher. This high level of PNC attendance could be explained by the fact that majority of mothers in our study population delivered at a health facility. Giving birth from a health facility by either normal vaginal delivery or caesarean section result in mothers spending at least 24 hours postpartum in the facility, hence more monitoring. This increases the chances of having PNC contacts with health care workers together with their babies. Findings from preliminary analysis further revealed that a bigger proportion of mothers was knowledgeable about postnatal care service and among those that did not seek 
PNC, the majority were not just aware. This means that knowledge about health service is crucial in influencing uptake. The presence of young mothers delivering from home and other places outside the formal health facilities points to reason to worry. It means that these young mothers much as they are very vulnerable to postpartum complications still consider giving birth from traditional birth attendants. Mothers also attended PNC because their babies needed immunization. Therefore uptake of PNC could be improved more by harmonising timing of PNC visits with immunization scheduled for babies in the 42 days following delivery.

From bivariate analysis, education level, employment status of mothers and their spouses, time spent at health facilities, amount spent on seeking PNC services and attending antenatal care were all significantly associated with uptake of PNC services. This is in agreement with findings from prior studies $(7,12-15)$. Education among women is associated increased awareness of health services and empowerment among them to take charge of their own health compared to uneducated women who tend to leave all decisions relate to their health and babies to their spouses.

From multivariate analysis, respondents who were self-employed and employed were 2.205 and 2.022 respectively more likely to use PNC services compared to those who were unemployed. Respondents whose husbands were employed were 4.099 more likely to use PNC services compared to those whose husbands were unemployed. Respondents who did not receive PNC services privately were 18.605 times more likely to use PNC services compared to those who received PNC services privately. Respondents who spent more than 5000 shillings in the process if PNC service utilization were 0.056 times less likely to use PNC services compared to those who spent less than 2000 shillings. Respondents who were married were 8.142 times less likely to use PNC services compared those who were single.

\section{CONCLUSION}

The general level of uptake of PNC services was 78.8\%. Being self-employed and employed, having employed husband and being married were all positively associated with uptake of PNC services among the study population. Receiving PNC service privately was negatively associated with uptake of PNC services while having to spend less than 2000 shillings in the due course of seeking PNC was positively associated with uptake of PNC services by young mothers (15-24 years) at Mukono general hospital.

\section{RECOMMENDATIONS}

The study, therefore, recommends that hence forth, government and partners should consider empowering citizens especially women economically through job creation initiatives, among others

\section{ACKNOWLEDGEMENTS}

We would like to thank the mothers who participated in the study and we acknowledge the contribution of Mukono General Hospital and lastly the Staff at Uganda Martyrs University, Faculty of Health Sciences.

\section{DECLARATION}

The authors declare that they had no conflict of interest. 


\section{REFERENCES}

[1]. WHO. WHO recommendations on postnatal care of the mother and newborn: World Health Organization; 2014.

[2]. WHO. WHO technical consultation on postpartum and postnatal care. World Health Organization; 2010.

[3]. WHO. Maternal mortality: World Health Organisation; 2019 [Available from: https://www.who.int/news-room/fact-sheets/detail/maternal-mortality.

[4]. WHO. Postnatal care for mothers and newborns: Highlights from the World Health Organization 2013 Guidelines. Avaible from: http://www who int/maternal_child_adolescent/publications/WHO-MCA-PNC-2014-Briefer_A4 pdf. 2015.

[5]. WHO. Newborns: reducing mortality: World Health Organisation; 2019 [Available from:https://www.who.int/news-room/fact-sheets/detail/newborns-reducing-mortality.

[6]. Motherhood S. Safe Motherhood: a matter of human rights and social justice. Retrieved July. 2002;10:2013.

[7]. Mon AS, Phyu MK, Thinkhamrop W, Thinkhamrop B. Utilization of full postnatal care services among rural Myanmar women and its determinants: a cross-sectional study. F1000Research. 2018;7.

[8]. Mosiur Rahman M, Haque SE, Sarwar Zahan M. Factors affecting the utilisation of postpartum care among young mothers in Bangladesh. Health \& social care in the community. 2011;19(2):138-47.

[9]. Neupane S, Doku D. Utilization of postnatal care among Nepalese women. Maternal and child health journal. 2013;17(10):1922-30.

[10].Dahiru T, Oche OM. Determinants of antenatal care, institutional delivery and postnatal care services utilization in Nigeria. Pan African medical journal. 2015;22(1).

[11].Hordofa MA, Almaw SS, Berhanu MG, Lemiso HB. Postnatal care service utilization and associated factors among women in Dembecha District, Northwest Ethiopia. Science Journal of Public Health. 2015;3(5):686-92.

[12]. Abraha TH, Gebrezgiabher BB, Aregawi BG, Belay DS, Tikue LT, Reda EB. Factors Associated with Compliance with the Recommended Frequency of Postnatal Care Services in Four Rural Districts of Tigray Region, North Ethiopia. Korean journal of family medicine. 2019;40(5):329.

[13].Akibu M, Tsegaye W, Megersa T, Nurgi S. Prevalence and determinants of complete postnatal care service utilization in northern Shoa, Ethiopia. Journal of pregnancy. 2018;2018.

[14].Larsen A, Cheyip M, Aynalem G, Dinh Th, Jackson D, Ngandu N, et al. Uptake and predictors of early postnatal follow-up care amongst mother-baby pairs in South Africa: Results from three population-based surveys, 2010-2013. Journal of global health. 2017;7(2).

[15].Jacobs C, Moshabela M, Maswenyeho S, Lambo N, Michelo C. Predictors of antenatal care, skilled birth attendance, and postnatal care utilization among the remote and poorest rural communities of Zambia: a multilevel analysis. Frontiers in Public Health. 2017;5:11.

[16].ML AN, Malonga F, Dramaix-Wilmet M, Donnen P. Determinants of maternal health services utilization in urban settings of the Democratic Republic of Congo-a case study of Lubumbashi City. BMC pregnancy and childbirth. 2012;12(1):66.

[17].Kanté AM, Chung CE, Larsen AM, Exavery A, Tani K, Phillips JF. Factors associated with compliance with the recommended frequency of postnatal care 
cognizancejournal.com

Kizito Omona et al, Cognizance Journal of Multidisciplinary Studies, Vol.1, Issue.3, March 2021, pg. 45-57 services in three rural districts of Tanzania. BMC pregnancy and childbirth. 2015;15(1):341.

[18]. Wilunda C, Oyerinde K, Putoto G, Lochoro P, Dall'Oglio G, Manenti F, et al. Availability, utilisation and quality of maternal and neonatal health care services in Karamoja region, Uganda: a health facility-based survey. Reproductive health. 2015;12(1):30.

[19].Rutaremwa G, Wandera SO, Jhamba T, Akiror E, Kiconco A. Determinants of maternal healthservices utilization in Uganda. BMC health services research. 2015;15(1):271.

[20].Kusasira D. Factors Influencing the uptake of Postnatal Care services Among Mothers: International Health Sciences University.; 2015.

[21].Izudi J, Amongin D. Use of early postnatal care among postpartum women in Eastern Uganda. International Journal of Gynecology \& Obstetrics. 2015;129(2):161-4.

[22].UNAIDS. HIV Prevention among adolescent girls and young women. Joint United Nations Programme on HIV/AIDS Geneva; 2016.

[23].Lisonkova S, Potts J, Muraca GM, Razaz N, Sabr Y, Chan W-S, et al. Maternal age and severe maternal morbidity: A population-based retrospective cohort study. PLoS medicine. 2017;14(5):e1002307.

[24].UBOS, ICF. Uganda Demographic and Health Survey 2016. Kampala, Uganda: UBOS and ICF; 2018.

[25].MOH. District Health Information System (DHIS 2) Mukono District. Ministry of Health; 2019.

[26].Ndugga P, Namiyonga NK, Sebuwufu D. Determinants of early postnatal care attendance: analysis of the 2016 Uganda demographic and health survey. BMC Pregnancy and Childbirth. 2020;20(1):1-14. 